\title{
Mitteilungen über kleine Planeten.
}

Photographic Observations at Winchester.

Planet I913 Gr.M.T. Position rgr3.o Magn. 498 Tokio Mar. 7 I $6^{\mathrm{h}} 3^{\mathrm{m}} \cdot \mathrm{O}$ I $1^{\mathrm{h}} 29^{\mathrm{m}} 19^{\mathrm{s}} \cdot 5+\mathrm{I} 7^{\circ}$ I $6^{\prime} \cdot 1$ I $2^{\mathrm{m}} \cdot 5$

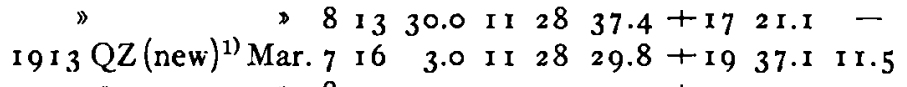
$\gg 81330.0$ II $2749.0+1943.7-$

Winchester, Mass., I 9 I 3 March 20 . Foel H. Metcalf.

1) Vgl. die Auffindung auf der Königstuhl-Sternw. A. N. 194.1 3 r. Red.

Aufnahmen auf der Königstuhl-Sternwarte. Planet Position I913.0 Tägl. Bew. Gr. Platte 1913 April 3 .

29 I Alice $\quad$ I $^{\mathrm{h}} 7^{\mathrm{m}} \cdot 8+0^{\circ} 5^{\prime}-0^{\mathrm{m}} \cdot 9+7^{\prime}$ I $3^{\mathrm{m}} \cdot 2$ B 3 I 79 I 95 Eurykleia $129.8-050-0.8+312.1$ \% 2 I I Isolda I $237.3-950-0.7+5$ II.7 A69 I 2 I 94 Prokne $1257.9+106-0.8+$ ro Ir.I B 3 I 8 I $579[1905 \mathrm{SD}]{ }_{13} 3.7+95^{1}-0.8+4$ II.8 1913 April 4.

I94 Prokne I $257.2+$ Io I6 -0.8+ I0 I I.I B 3 I 83 I9 I 3 RE (neu) I 3 I. 6 + I3 $3-0.8+6$ I 3.2 579 [1905 SD) $132.9+954-0.8+3$ 1 3.8 》

Platte m.Z. Kgst. Plattenmitte Beob.

A $69 \mathrm{I} 2$ I I ${ }^{\mathrm{h}} \mathrm{I}^{\mathrm{m}} \mathrm{I}$ I $\mathbf{2}^{\mathrm{h}} \mathrm{I}^{\mathrm{m}}-\mathrm{I}^{\circ} A$. Massinger

$\mathrm{B} 3179$ 10 $25.3 \quad 1247-9 \quad F$. Kaiser $\begin{array}{rrrrrr}3181 & 12 & 30.7 & 12 & 54+10 \\ 3183 & 9 & 44.8 & 13 & 0 & +11\end{array}$

Königstuhl-Sternw., Heidelberg, I 9 г 3 April 5. M. Wolf.

Beobachtungen auf der k. k. Sternwarte Wien. I 90 Ismene I 9 I 3 März 3 I I I ${ }^{\mathrm{h}} 43^{\mathrm{m}}$ M.E.Z. I $3^{\mathrm{h}} 3^{2^{\mathrm{m}}} 5^{\mathrm{s}}-6^{\circ}{ }_{\text {I }}{ }^{\prime} \cdot 3$ 26 I Prymno "April 4 I0 16》I3 I6 21 - 652.7 312 Pierretta 306 Unitas 》 März 28 Korr.d.Eph.(B.J.I915) $-0^{m} .4+4^{\prime}$ 364 Isara "März 28 " " " " - $0.9+5$ 6 I5 [1906 VR] steht am Ort der Ephemeride.

Wien, I9 3 April 5.

\section{F. Rheden.}

Elemente und Ephemeride von 19I3 QW. geleitet.

Die Bahn ist aus Wien Febr. 26, März 14 und 28 ab-

$$
\begin{aligned}
& \text { Epoche I } 9 \text { I } 3 \text { März I } 4.5 \text { m. Z. Berlin. }
\end{aligned}
$$

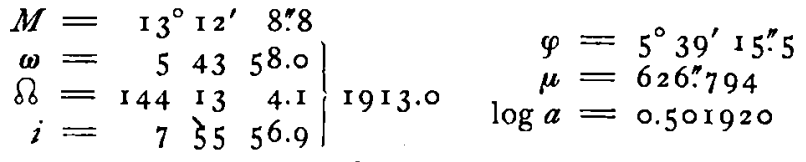

$$
\begin{aligned}
& \text { Ephemeride für } \mathrm{I}^{\mathrm{h}} \mathrm{m} . \mathrm{Z} \text {. Berlin. Gr. } \mathrm{I}^{\mathrm{m}} \text {. } \\
& \begin{array}{llll}
1913 & \alpha & \delta & \log \Delta
\end{array} \\
& \begin{array}{lll}
\text { März } 29 \quad{ }^{\mathrm{h}}{ }^{\mathrm{h}} \mathrm{O}^{\mathrm{m}} \cdot 6+\mathrm{I}^{\circ}{ }_{4} 0^{\prime} & 0.294
\end{array} \\
& \begin{array}{llllll}
\text { April } 6 \quad \text { I0 } & 47.3 & \text { I3 } & 10 & 0.308
\end{array} \\
& \begin{array}{lllll}
14 & 10 & 45.4 & 1329 & 0.323
\end{array} \\
& 22 \text { I0 } 44.8 \quad \text { I } 337 \quad 0.340 \\
& \begin{array}{lllll}
30 & \text { I0 } 45.6 & \text { I } 3 & 35 & 0.35^{8}
\end{array}
\end{aligned}
$$

\begin{tabular}{|c|c|c|c|c|c|c|c|c|}
\hline & & \multirow{2}{*}{\multicolumn{3}{|c|}{$\alpha$ app. }} & & & \multirow{3}{*}{$\begin{array}{c}\log r \\
0.7541\end{array}$} & \multirow{4}{*}{$\begin{array}{c}\log \Delta \\
0.675 \\
0.673\end{array}$} \\
\hline \multirow{2}{*}{\multicolumn{2}{|c|}{\begin{tabular}{ll}
\multicolumn{1}{r}{${ }^{1913}$} \\
April & 2
\end{tabular}}} & & & & \multicolumn{2}{|c|}{ app. } & & \\
\hline & & I $4^{b}$ & $13^{\mathrm{m}}$ & $2^{s}$ & $-6^{\circ}$ & I.'0 & & \\
\hline & 6 & I 4 & I I & 6 & 6 & 14.3 & 0.7539 & \\
\hline & 10 & 14 & 9 & 5 & 6 & 9.8 & 0.7537 & 0.6713 \\
\hline & I 4 & I 4 & 7 & 0 & 6 & $5 \cdot 3$ & 0.7534 & 0.6699 \\
\hline & I 8 & 14 & 4 & $5 \mathrm{I}$ & 6 & 0.9 & 0.7532 & 0.6690 \\
\hline & 22 & 14 & 2 & 42 & 5 & 56.8 & $0.753^{\circ}$ & 0.6685 \\
\hline & 26 & I 4 & $\circ$ & 33 & 5 & 53.0 & 0.7527 & 0.6685 \\
\hline & 30 & I 3 & $5^{8}$ & 24 & 5 & $49 \cdot 7$ & $0.75^{2} 5$ & 0.6690 \\
\hline \multirow[t]{7}{*}{ Mai } & 4 & I 3 & $5^{6}$ & 18 & 5 & 46.9 & 0.7523 & 0.6699 \\
\hline & 8 & I 3 & 54 & 14 & 5 & 44.7 & 0.7520 & 0.6712 \\
\hline & I 2 & 13 & $5^{2}$ & r 6 & 5 & $43 \cdot I$ & $0.75 \times 8$ & 0.6729 \\
\hline & I 6 & I 3 & 50 & 24 & 5 & 42.3 & 0.7516 & $0.675^{1}$ \\
\hline & 20 & 13 & 48 & 37 & 5 & 42.3 & 0.75 I 3 & 0.6776 \\
\hline & 24 & I 3 & 46 & $5^{8}$ & 5 & 43.0 & $0.75 \mathrm{II}$ & 0.6804 \\
\hline & 28 & I 3 & 45 & 28 & 5 & $44 \cdot 5$ & 0.7508 & $0.683^{6}$ \\
\hline \multirow[t]{5}{*}{ Juni } & I & I 3 & 44 & 7 & 5 & $47 \cdot 3$ & 0.7506 & $0.687 \mathrm{I}$ \\
\hline & 5 & I 3 & 42 & 55 & 5 & 50.8 & 0.7503 & 0.6908 \\
\hline & 9 & I 3 & 41 & 53 & 5 & $55 \cdot 3$ & $0.750 \mathrm{I}$ & 0.6948 \\
\hline & I 3 & I 3 & $4 I$ & I & 6 & 0.7 & 0.7498 & 0.6990 \\
\hline & I 7 & I 3 & 40 & 20 & -6 & 6.0 & 0.7496 & 0.7034 \\
\hline
\end{tabular}

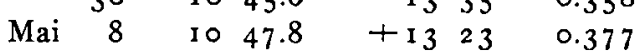

Ephemeride des Planeten 6i7 Patroclus gerechnet für $\mathbf{I}^{\mathrm{h}} \mathrm{m}$. Z. Berlin mit den Elementen B. J. I 9 I 4 .

Prag, I9I3 April 2.

Einige Identifizierungen von Planeten.

$1906 \mathrm{TV}$ ist $28 \mathrm{I}$ Lucretia, s. A. N. I 7I.Io. Der daselbst und 171.45, 174.291 als Lucretia bezeichnete Planet war ein neuer. Dieser wird mit $1906 \mathrm{TV}^{\mathrm{a}}$ bezeichnet.

I $909 \mathrm{JF}$ ist 302 Clarissa (A. N. I83.19I); die Elemente sind jetzt verbessert.

1908 CA (Metcalf A. N. 177.95) wird durch eine provisorische Bahn von $19 \times 3 \mathrm{QV}$ fast genau dargestellt. In $\delta$ bleiben nur 2', weitere Beobachtungen von $Q V$ werden über die Identität bald entscheiden.

Von den A requipa-Planeten (A. N. 179.209) ist Nr. $3=566$ Stereoskopia $=1899 \mathrm{JF}$. Nr. 7 ist $=70 \mathrm{I}$ [1910KN]; mit $\mathrm{d} M=+\mathrm{r} 5^{\prime}$ werden die Beobachtungen 1899 Juni $12,13,14, I_{5}$ auf $+1^{s},-4^{s},-3^{s}, o^{s}$ in $\alpha$ und $-r^{\prime} 8,-2 \cdot 7, \quad-2 \cdot 8, \quad-r^{\prime} 8$ in $\delta$ dargestellt. Nr. $I_{5}$ ist 48 I Emita. Die Elemente im B. J. I 915 stellen mit d $M$ $=+39^{\prime}$ die Beobachtung 1899 Juli 7 fast völlig dar. 707 [1910 LD] ist anscheinend nicht unter den ArequipaPlaneten, wenigstens nicht $=\mathrm{Nr}$. 6 oder Nr. 19. Auch $35^{8}$ Apollonia ist nicht darunter, die Notiz A. N. 179.210. ist also hinfällig.

Ferner erweist sich jetzt nach Ergänzung der Störungsrechnung von 462 Eriphyla die früher angenommene Identität dieses Planeten mit einem 1892 Januar 19 in Paris photographierten Objekte (A. N. 155.26) als ausgeschlossen. Mit einer Korrektion von $\mu$ in meinen Elementen im B. J. I 915 um < 0.2 werden alle Erscheinungen von 1896 bis I 9 1 2, also 1 896-97, 1900, 02, 05, 07, 09, 1 2 auf wenige Bogenminuten dargestellt, während 1892 über $2^{\circ}$ in Länge abweicht.

Berlin-Dahlem, I 9 I 3 März 27.

Inhalt zu Nr. 4643. E. Fost. Beobachtungen von veränderlichen Sternen. I93. - M.Viljev. Ephemeride des Kometen I852 IV ( Westphal) für 1913. 203. - F. Alker. Beobachtungen über die Trübung der Atmosphäre. 205. - F. P. Lagrula. 233 Asterope. 205. Mitteilungen uber kleine Planeten. 207. 